\title{
EDITORIAL
}

\section{Follicular helper T cells in immune homeostasis}

\author{
Cellular \& Molecular Immunology (2012) 9, 367-368; doi:10.1038/cmi.2012.27; published online 3 September 2012
}

I $\mathrm{n}$ recent years, follicular helper $\mathrm{T}\left(\mathrm{T}_{\mathrm{FH}}\right)$ cells have become a hot topic for immunologists due to their pivotal role in modulating antibody-mediated immune responses. Although helper $\mathrm{T}\left(\mathrm{T}_{\mathrm{H}}\right) 2$ cells have long been known to provide help for $\mathrm{B}$-cell responses, $\mathrm{T}_{\mathrm{FH}}$ cells have been identified only recently as a distinct lineage of $\mathrm{T}_{\mathrm{H}}$ cells that is specific for the provision of help to $\mathrm{B}$ cells to generate antibody responses. $\mathrm{T}_{\mathrm{FH}}$ cells are distinguished by their unique surface marker expression (CXCR $5^{\text {high }}, \mathrm{PD}-1^{\text {high }}$, ICOS ${ }^{\text {high }}$ and CCR $7^{\text {low }}$ ), cytokine profile (IL-21 and IL-6) and, more importantly, transcriptional factor expression (Bcl6). Furthermore, $\mathrm{T}_{\mathrm{FH}}$ cells are endowed with the capacity to migrate to B-cell follicles in secondary lymphoid organs and closely interact with B cells to promote the formation of germinal centers (GCs). The stable cognate interaction between $\mathrm{T}_{\mathrm{FH}}$ and $\mathrm{B}$ cells in the GC is mutually beneficial to them for their development and function. Specifically, $\mathrm{T}_{\mathrm{FH}}$ cells help B cells produce long-lived memory B cells and high-affinity antibodies and, in reciprocation, they are helped by B cells for further differentiation through cell cognation. This is how effective antibody responses are generated to provide protection against pathogenic microbes.

In this issue, there are three reviews to update the study on the biology of $\mathrm{T}_{\mathrm{FH}}$ cells with a focus on the involvement of these cells in disease settings. ${ }^{1-3}$ Chen et al. ${ }^{1}$ provide an overview of the up-to-date knowledge on ontological and physiological properties of $\mathrm{T}_{\mathrm{FH}}$ cells. Shekhar and Yang ${ }^{2}$ have reviewed the current literature on the role of $\mathrm{T}_{\mathrm{FH}}$ cells in pathologies, including autoimmunity and immunodeficiency; whereas, Kemeny ${ }^{3}$ discusses the contribution of $\mathrm{T}_{\mathrm{FH}}$ cells to the pathogenesis of allergic diseases. The function of $\mathrm{T}_{\mathrm{FH}}$ cells is beneficial to the host in that it helps establish the humoral arm of adaptive immunity to fight against the invasion of pathogens. Although the benefit is obvious, the 'dark' side of these cells has also started to be recognized. In addition to the significant progress in $\mathrm{T}_{\mathrm{FH}}$ cell biology, including cellular and molecular characterization, distribution and mechanistic study on signaling and antibody class switching, recent studies on $\mathrm{T}_{\mathrm{FH}}$ cells have now expanded to various disease settings, such as autoimmune diseases, immunodeficiency, lymphoma, asthma and other allergic diseases. The studies on autoimmunity are extensively performed in various types of lupus-prone mouse models with considerable amount of work on human patients. The data on allergic diseases are mostly from immunoglobulin $\mathrm{E}$ ( IgE) responses, especially via the involvement of IL-21.

Correspondence: Dr X Yang Laboratory for Infection and Immunity, Department of Medical Microbiology, Faculty of Medicine, University of Manitoba Winnipeg,

Manitoba, Canada.

E-mail: yangxi@cc.umanitoba.ca

Received 5 July 2012; accepted 9 July 2012
The contribution of $\mathrm{T}_{\mathrm{FH}}$ cells in the development of autoimmunity and allergic diseases suggests the importance of a balanced response of this T-cell subset in maintaining immune homeostasis. However, the mechanism for maintaining this balance remains largely unclear. The identification of follicular regulatory $\mathrm{T}\left(\mathrm{T}_{\mathrm{FR}}\right)$ cells, which are involved in the regulation of $\mathrm{T}_{\mathrm{FH}}$ cell development and function, has shed some light on this field. ${ }^{4,5}$ Notably, $\mathrm{T}_{\mathrm{FR}}$ cells express FoxP3 and CTLA-4 and produce IL-10, like classic regulatory $\mathrm{T}\left(\mathrm{T}_{\mathrm{REG}}\right)$ cells, but they depend on Bcl6 for their development and express CXCR5, as do classic $\mathrm{T}_{\mathrm{FH}}$ cells. These FoxP3 ${ }^{+} \mathrm{T}_{\mathrm{FR}}$ cells are found to inhibit GC responses, preventing exaggerated GC maturation and antibody development. It is likely that at least a portion of the lupus-prone mice and humans have deficiencies in the development and/or function of $\mathrm{T}_{\mathrm{FR}}$ cells. Moreover, the identification of $\mathrm{CD} 8{ }^{+} \mathrm{T}_{\mathrm{REG}}$ cells in $\mathrm{GC}$ and the modulating effect of these cells on $\mathrm{T}_{\mathrm{FH}}$ cell development and function emphasizes on the importance of networking of different cell types in maintaining the homeostasis of the immune system. ${ }^{6}$ In addition to the lack of regulation by $\mathrm{T}_{\mathrm{FR}}$ cells, intrinsic hyper $\mathrm{T}_{\mathrm{FH}}$ cell responses may exist in the individuals with autoimmunity, which may be involved in Bcl6 and Blimpl dysregulation. By the same token, the deficiency in $\mathrm{T}_{\mathrm{FH}}$ cells in some immunodeficiency diseases may also be related to the imbalance of these transcriptional factors. Further study on the balance of $\mathrm{T}_{\mathrm{FH}}$ and $\mathrm{T}_{\mathrm{FR}}$ cells especially in the setting of $\mathrm{B}$-cell follicle and GC appears to be crucial for understanding the pathogenesis, and development of new therapeutic approaches to these diseases.

The studies on the role of $\mathrm{T}_{\mathrm{FH}}$ cells in allergic diseases are mainly based on the responses of IgE antibody, which is the typical antibody isotype mediating allergic diseases including allergic asthma. ${ }^{2}$ It has been long recognized that IL-4 and CD40-CD40L interactions are critical for IgE responses. $\mathrm{T}_{\mathrm{FH}}$ cells can produce IL-4 and express CD40L, which are sufficient to promote IgE responses. However, recent studies suggest that IL-21, the most typical cytokine of $\mathrm{T}_{\mathrm{FH}}$ cells, is critical for IgE responses. In fact, it appears that IL-21 plays a critical role in $\mathrm{T}_{\mathrm{FH}}$ cell-mediated help for B cells in somatic mutation and isotype class switching. Given the fact that class switching for different antibody isotypes requires different types of cytokines, i.e., IL-4 for IgE, interferon- $\gamma$ for IgG2a, transforming growth factor- $\beta$ and IL- 6 for IgA, it is unlikely that a single $\mathrm{T}_{\mathrm{FH}}$ cell can produce all of the different types of cytokines. This raises a question if $\mathrm{T}_{\mathrm{FH}}$ cells consist of different subsets which have common features of $\mathrm{T}_{\mathrm{FH}}$ cells, particularly IL-21 production, but exhibit differential $\mathrm{T}_{\mathrm{H}} 1, \mathrm{~T}_{\mathrm{H}} 2$ and $\mathrm{T}_{\mathrm{REG}}$ cell cytokine patterns. This is an important question to address in future studies, not only for allergic diseases, but also for infectious diseases, considering the significant difference of the various isotypes of antibody in pathogen neutralization, opsonization and antibody-dependent cytotoxicity. 
Notably, but not surprisingly, most of the mechanistic studies on $\mathrm{T}_{\mathrm{FH}}$ cells in disease settings are performed in animal models, particularly the mouse. Considering the functional site of $\mathrm{T}_{\mathrm{FH}}$ cells, GC, it is rather difficult to perform studies focusing on local responses in humans. The human studies so far are virtually all from studies on circulating $\mathrm{T}_{\mathrm{FH}}\left(\mathrm{cT}_{\mathrm{FH}}\right)$ cells. Interestingly, $\mathrm{cT}_{\mathrm{FH}}$ cells, which are identified in a study involving systemic lupus erythematosus patients, express CXCR5, ICOS and PD-1, but not BCL6 and IL-21. ${ }^{7}$ Given that BCL6 and IL-21 are the most critical transcription factor and cytokine for $\mathrm{T}_{\mathrm{FH}}$ cell development and function, it is still debatable as to what degree the $\mathrm{cT}_{\mathrm{FH}}$ cells represent $\mathrm{GC} \mathrm{T}_{\mathrm{FH}}$ cells. Further study on the correlation and difference between $\mathrm{T}_{\mathrm{FH}}$ cells in peripheral blood and secondary lymphoid organs is needed.

Recent studies have provided significant evidence on the role of $\mathrm{T}_{\mathrm{FH}}$ cells in protective and pathological immune responses, although much remains to be known. $\mathrm{T}_{\mathrm{FH}}$ cells play a critical role in eliciting effective antibody responses during vaccination or infection; whereas, dysregulation of these cells can lead to pathologies, such as autoimmunity and allergy. However, many questions remain concerning the mechanisms of how the function of $\mathrm{T}_{\mathrm{FH}}$ cells is regulated to avoid development of diseases and maintain immune homeostasis. What are the factors that trigger $\mathrm{T}_{\mathrm{FH}}$ cell differentiation? What are the molecular events that result in dysregulation of $\mathrm{T}_{\mathrm{FH}}$ cells? How do $\mathrm{T}_{\mathrm{FR}}$ cells and other regulatory cells interact with $\mathrm{T}_{\mathrm{FH}}$ cells to maintain humoral immune tolerance? Are there subsets of $\mathrm{T}_{\mathrm{FH}}$ cells that are destined to perform different functions including isotype switches? Are $\mathrm{cT}_{\mathrm{FH}}$ cells indeed related to classic $\mathrm{GC} \mathrm{T}_{\mathrm{FH}}$ cells? Can $\mathrm{T}_{\mathrm{FH}} / \mathrm{cT}_{\mathrm{FH}}$ cells be used as diagnostic and monitoring markers for diseases like lymphoma? To what extent the findings from mouse studies can be relevant to humans? Future studies need to answer these questions, which may have implications for designing the prophylactic and/or therapeutic strategies against these diseases.

Xi Yang

Laboratory for Infection and Immunity, Department of Medical Microbiology, Faculty of Medicine, University of Manitoba, Winnipeg, Manitoba, Canada

\section{ACKNOWLEDGEMENTS}

$\mathrm{XY}$ is Canada Research Chair in Infection and Immunity. This work was supported by grants from Canadian Institutes of Health Research and Manitoba Health Research Council.

1 Chen M, Guo Z, Ju W, Ryffel B, He X, Zheng SG. The development and function of follicular helper T cells in immune responses. Cell Mol Immunol 2012; in press

2 Shekhar S, Yang X. The darker side of follicular helper T cells: from autoimmunity to immunodeficiency. Cell Mol Immunol, 2012; in press

3 Kemeny DM. The role of T follicular helper cells in allergic diseases. Cell Mol Immunol 2012; in press

4 Linterman MA, Pierson W, Lee SK, Kallies A, Kawamoto S, Rayner TF et al. Foxp3 ${ }^{+}$ follicular regulatory T cells control the germinal center response. Nat Med 2011; 17: 975-982.

5 Chung Y, Tanaka S, Chu F, Nurieva RI, Martinez GJ, Rawal S et al. Follicular regulatory $\mathrm{T}$ cells expressing Foxp3 and Bcl-6 suppress germinal center reactions. Nat Med 2011; 17: 983-988.

6 Kim HJ, Verbinnen B, Tang X, Lu L, Cantor H. Inhibition of follicular T-helper cells by $\mathrm{CD}^{+}$regulatory T cells is essential for self tolerance. Nature 2010; 467: 328-332.

7 Simpson N, Gatenby PA, Wilson A, Malik S, Fulcher DA, Tangye SG et al. Expansion of circulating $T$ cells resembling follicular helper $T$ cells is a fixed phenotype that identifies a subset of severe systemic lupus erythematosus. Arthritis Rheum 2010; 62: $234-244$. 